\title{
ON THE ARITHMETIC OF TUBE DOMAINS (BLOWING-UP OF THE POINT AT INFINITY)
}

\author{
BY I. SATAKE
}

The purpose of this talk is to construct, in a certain canonical manner, a "blow-up" of the isolated singularity which appears as a point at infinity of an arithmetic quotient of a symmetric tube domain. Similar (but slightly different) blowing-ups have already appeared in some special cases in the works of Pyatetskii-Shapiro, Igusa [4] (Siegel modular case) and Hirzebruch [3] (Hilbert modular case). It should be possible to extend our construction to the case of general symmetric domains via their realizations as "Siegel domains of the third kind" (cf. [1], [6], [7b]). But, for the sake of simplicity, we shall here restrict ourselves to the simplest case. ${ }^{1}$

1. Let $U$ be an $n$-dimensional real vector space endowed with a (positive-definite) inner product $\langle>$ and $\Omega$ a (nonempty) open convex cone in $U$ with its vertex at the origin of $U$. We assume that $\Omega$ does not contain any straight line (not necessarily passing through the origin). Let $G_{0}$ be the identity connected component of the (linear) automorphism group

$$
\operatorname{Aut}(\Omega)=\{g \in \operatorname{GL}(U) \mid g \Omega=\Omega\} .
$$

In the following, we assume $\Omega$ to be "homogeneous" and "self-dual"; these mean that $G_{0}$ is transitive on $\Omega$ and that $\Omega$ coincides with its dual

$$
\Omega^{*}=\left\{u \in U \mid\left\langle u, u^{\prime}\right\rangle>0 \text { for all } u^{\prime} \in \bar{\Omega}-\{0\}\right\},
$$

where $\bar{\Omega}$ denotes the closure of $\Omega$. It is known [9] that the latter condition is equivalent to saying that ${ }^{t} G_{0}=G_{0}, t$ denoting the adjoint with respect to the inner product $\left\langle>\right.$, and this implies that $G_{0}$ is the identity connected

An expanded version of an invited address delivered at the 698th meeting of the Society in La Jolla, California on November 18, 1972; received by the editors May 7, 1973. I wish to express here my thanks to Mumford, Hartshorne, and Yamaguchi for very helpful comments on my original manuscript.

AMS (MOS) subject classifications (1970). Primary 32N15, 10E99, $14 \mathrm{M} 20$.

Key words and phrases. Self-dual cones, arithmetic groups, analytic spaces.

${ }^{1}$ After the talk I have learned, by a written communication with Mumford, that a closely related theory has been developed by Demazure, Mumford and others; compare his recent seminar notes at Harvard [5]; cf. also M. Demazure, Sous-groupes algebriques de rang maximum du groupe de Cremona, Ann. Sci. Ecole Norm. Sup 3 (1970), and M. Hochster, Rings of invariants of tori, Cohen-Macaulay rings generated by monomials and polytopes, Ann. of Math.96 (1972). It seems that a fair part of our results overlaps with theirs obtained in a much wider framework. Some connections with their theory are alluded to in $\S 4$ and $\S 9$ of the text. 
component of a reductive (real) algebraic group $G$. We fix a lattice $M$ in $U$ and assume that $\Omega$ is "rational" with respect to $M$, meaning that when we express $G$ as a matrix group with respect to a basis of $M, G$ is defined by polynomial equations with coefficients in $\boldsymbol{Q}$ (the field of rational numbers). Under this assumption the inner product in $U$, for which $\Omega$ is self-dual, can also be chosen to be rational, i.e. in such a way that the dual lattice $M^{*}$ of $M$ is commensurable with $M$ itself. Finally, we assume that $\Omega$ is " $Q$-anisotropic". This means that there are no rational points on the boundary of $\Omega$ except the origin. It is known [2] that this condition is equivalent to saying that the $Q$-rank of $G$ is $=1$. This implies that the maximal $Q$-split torus in $G$ is given by the group of dilatations $u \mapsto \lambda u$ $\left(\lambda \in \boldsymbol{R}^{\times}\right)$and the semisimple part of $G$ is $\boldsymbol{Q}$-simple and $\boldsymbol{Q}$-compact. A complete list of self-dual cones satisfying these conditions is available from the classification theory of simple algebraic groups.

ExAmple. Let $U=\boldsymbol{R}^{n}, M=\boldsymbol{Z}^{n}$, and let $S=\left(a_{i j}\right)$ be an $n \times n$ nonsingular rational symmetric matrix with signature $(1, n-1)$ such that $a_{11}>0$ and the corresponding quadratic form $S(x)={ }^{t} x S x(x \in U)$ does not express zero nontrivially in $\boldsymbol{Q}^{n}$. (Hence $n \leqq 4$.) Then, the quadratic cone

$$
\Omega=\left\{x=\left(x_{i}\right) \in \boldsymbol{R}^{n} \mid S(x)>0, x_{1}>0\right\}
$$

is self-dual with respect to the inner product $\langle x, y\rangle={ }^{t} x S_{0} y$ where $S_{0}$ is an $n \times n$ positive-definite symmetric rational matrix such that $\left(S S_{0}^{-1}\right)^{2}=$ 1 , and all the above conditions are satisfied for this setting.

2. Let $\Omega$ be an open convex cone in $U$ satisfying all the conditions mentioned in $\S 1$. Let $U_{C}$ be the complexification of $U$ and consider the tube domain $U+i \Omega$ in $U_{C}$, which is an (unbounded) symmetric domain. The semidirect product $\tilde{G}_{0}=G_{0} \cdot U$ then acts transitively on $U+i \Omega$ by

$$
g=g u_{1}: u \mapsto g\left(u+u_{1}\right) \quad\left(g \in G_{0}, u_{1} \in U\right) .
$$

Let $\Gamma$ be an "arithmetic" subgroup of $G_{0}$ leaving $M$ fixed, i.e. a subgroup of finite index of $\left\{g \in G_{0} \mid g M=M\right\}$. Then the semidirect product $\tilde{\Gamma}=\Gamma \cdot M$ acts on $U+i \Omega$ properly discontinuously. When $\Gamma$ has no element of finite order other than the identity element, the quotient space $\tilde{\Gamma} \backslash(U+i \Omega)$ is a (nonsingular) complex manifold.

From the theory of compactification [1], [6], [7a] it is known that the quotient space $\tilde{\Gamma} \backslash(U+i \Omega)$ can be completed (locally) to a normal analytic space $\tilde{\Gamma} \backslash(U+i \Omega) \cup\{\infty\}$ by adjoining a single point at infinity, which turns out to be a singular point except for some lower dimensional cases. It is an important problem in the theory of automorphic functions to construct a (canonical) desingularization, or at least a nice blow-up, of the singularities of this kind (locally and globally). A possible approach 
to this problem will consist of studying the ring of automorphic forms and then performing the (iterated) "monoidal transformation" with respect to the ideal of cusp forms, as was done by Igusa [4] for the case of (congruence) Siegel modular groups. In this lecture we shall present a more direct geometric approach to the problem.

3. For $m \in M^{*}$, we put

$$
\zeta_{m}(u)=\exp (2 \pi i\langle m, u\rangle) \quad\left(u \in U_{c}\right)
$$

where $\langle>$ denotes the natural $(\boldsymbol{C}$-bilinear) extension of the given inner product in $U$. For convenience, we fix a numbering of the points in $\Omega \cap M^{*}$ :

$$
\Omega \cap M^{*}=\left\{m^{(j)} \mid j=1,2, \ldots\right\}
$$

and write $\zeta^{(j)}$ for $\zeta_{m^{(j)}}$. Consider a map $\phi$ of $U+i \Omega$ into an infinitedimensional projective space $P_{\infty}(C)$ defined by

$$
\phi(u)=\left(\ldots, \zeta^{(j)}(u), \ldots\right) .
$$

Clearly this mapping gives rise to an injection of the quotient space $(U+i \Omega) / M$ into $P_{\infty}(C)$. Our first aim is to determine the structure of the set of limit points at infinity obtained from this imbedding. Since $P_{\infty}(C)$ is not compact (not even locally compact), we have to define such limit points in some restricted sense.

For that purpose, let

$$
\vec{l}=\left\{\lambda u_{1} \mid \lambda>0\right\} \quad\left(u_{1} \in \Omega\right)
$$

be a half-line contained in $\Omega$. A point $m^{\left(j_{1}\right)}$ is called $\vec{l}$-minimal if $\left\langle m^{(j)}, u_{1}\right\rangle$ $\left(m^{(j)} \in \Omega \cap M^{*}\right)$ attains its minimum at $m^{(j)}=m^{\left(j_{1}\right)}$. Let $\sigma(\vec{l})$ be the convex closure of the set of all $\vec{l}$-minimal elements in $\Omega \cap M^{*}$. Then $\sigma(\vec{l})$ is a finite cell (= convex polygon) and all points in $\sigma(\vec{l}) \cap M^{*}$ are $\vec{l}$-minimal. We denote by $\Sigma=\Sigma\left(\Omega, M^{*}\right)$ the collection of all cells $\sigma=\sigma(\vec{l})$ obtained in this way for $\vec{l} \subset \Omega$, and by $|\Sigma|$ the set-theoretical union of all $\sigma \in \Sigma$. Then, from the assumptions on $\Omega$, it is easy to see that $|\Sigma|$ coincides with the boundary of the convex closure of $\Omega \cap M^{*}$, and $\Sigma$ is a locally finite cell complex such that every cell is a face of some $(n-1)$-dimensional cell. $|\Sigma|$ is called (in Japanese) a "Hariko" of $\Omega \cap M^{*}$.

To each $\sigma$ on the Hariko, we want to attach a certain projective variety. We put $J(\sigma)=\left\{j \mid m^{(j)} \in \sigma\right\}$.

LEMMA 1. Suppose we have a linear relation

$$
\sum_{j \in J(\sigma)} c_{j} m^{(j)}=0
$$

\footnotetext{
2 "Hariko" is a name of a Japanese handicraft to produce various figures (such as dolls, pets, tigers) by pasting pieces of paper.
} 
Then we have

$$
\sum_{j \in J(\sigma)} c_{j}=0 .
$$

In fact, let $\operatorname{dim} \sigma=r$ and let $\left\{m^{\left(i_{0}\right)}, \ldots, m^{\left(i_{r}\right)}\right\}$ be a maximal set of independent points in $\sigma \cap M^{*}$. Then, since the affine subspace spanned by $\sigma$ does not contain the origin of $U$, the vectors $m^{\left(i_{0}\right)}, \ldots, m^{\left(i_{r}\right)}$ are linearly independent. Hence every $m^{(j)} \in \sigma \cap M^{*}$ can be expressed uniquely in the form

$$
m^{(j)}=\sum_{k=0}^{r} a_{j k} m^{\left(i_{k}\right)}
$$

with $a_{j k} \in \boldsymbol{R}$ and $\sum_{k=0}^{r} a_{j k}=1$. Hence the relation (2) implies $\sum_{j \in J(\sigma)} c_{j} a_{j k}=$ 0 , for $0 \leqq k \leqq r$, whence we get (3).

In the relation (2) we may assume $c_{j} \in Z$, since $m^{(j)}$ 's belong to a lattice. Then we can consider the corresponding monomial equation in the indeterminates $\xi_{j}(j \in J(\sigma))$ which is homogeneous by virtue of $(3)$ :

$$
\prod_{+} \xi_{j}^{c_{j}}=\prod_{-} \xi_{j}^{\left|c_{j}\right|}
$$

where $\prod_{+}$(resp. $\left.\prod_{-}\right)$denotes the product taken over all $j \in J(\sigma)$ such that $c_{j}>0$ (resp. $c_{j}<0$ ). We first define an affine variety $A_{\sigma} \subset P_{\infty}(C)$ as the set of all $\left(\xi_{j}\right) \in P_{\infty}(C)$ satisfying the condition

$$
\xi_{j}=0 \Leftrightarrow j \notin J(\sigma)
$$

along with all the equations $\left(2^{\prime}\right)$ obtained from the integral linear relations (2). We also denote by $M(\sigma)$ the submodule of $M^{*}$ generated by $\left\{m^{\left(j^{\prime}\right)}-m^{(j)} \mid j, j^{\prime} \in J(\sigma)\right\}$ and by $\operatorname{Hom}\left(M(\sigma), C^{\times}\right)$the (multiplicative) group of all homomorphisms $\theta: M(\sigma) \rightarrow C^{\times}$, where $C^{\times}$is the (multiplicative) group of all nonzero complex numbers. Then we have

Lemma 2. $A_{\sigma} \approx \operatorname{Hom}\left(M(\sigma), C^{\times}\right) \approx\left(C^{\times}\right)^{r}$ where $r=\operatorname{dim} \sigma$.

In fact, every $m \in M(\sigma)$ can be written in the form

$$
m=\sum_{j \in J(\sigma)} b_{j}\left(m^{(j)}-m^{(j o)}\right)
$$

with $b_{j} \in \boldsymbol{Z}$, where $j_{0}$ is a fixed element in $J(\sigma)$. Given $x=\left(\xi_{j}\right) \in A_{\sigma}$, we set

$$
\theta_{x}(m)=\prod_{j \in J(\sigma)}\left(\xi_{j} / \xi_{j_{0}}\right)^{b_{j}} .
$$

Then, by virtue of $\left(2^{\prime}\right), \theta_{x}(m)$ is well-defined though the expression (5) may not be unique. Clearly, $\theta_{x} \in \operatorname{Hom}\left(M(\sigma), C^{\times}\right)$and the correspondence $x \mapsto \theta_{x}$ is continuous. To show that this mapping is actually bijective, 
take a basis $\left(m_{1}, \ldots, m_{r}\right)$ of $M(\sigma)$ and write

$$
\left\{\begin{aligned}
m_{i} & =\sum_{j \in J(\sigma)} b_{i j}\left(m^{(j)}-m^{\left(j_{0}\right)}\right) & & (1 \leqq i \leqq r), \\
m^{(j)}-m^{\left(j_{0}\right)} & =\sum_{k=1}^{r} a_{j k} m_{k} & & (j \in J(\sigma)),
\end{aligned}\right.
$$

where $b_{i j}, a_{j k} \in \boldsymbol{Z}$, and $a_{j k}$ 's are uniquely determined. Given $\theta \in$ $\operatorname{Hom}\left(M(\sigma), C^{\times}\right)$, put $x_{\theta}=\left(\xi_{j}\right)$, where

$$
\xi_{j}= \begin{cases}\prod_{k=1}^{r} \theta\left(m_{k}\right)^{a_{j k}} & \text { for } j \in J(\sigma), \\ 0 & \text { for } j \notin J(\sigma) .\end{cases}
$$

Then it is immediate that $x_{\theta} \in A_{\sigma}$. From (6) one has

so that by $\left(2^{\prime}\right)$

$$
m^{(j)}-m^{\left(j_{0}\right)}=\sum_{k=1}^{r} \sum_{j^{\prime} \in J(\sigma)} a_{j k} b_{k j^{\prime}}\left(m^{\left(j^{\prime}\right)}-m^{\left(j_{0}\right)}\right)
$$

$$
\xi_{j} / \xi_{j_{0}}=\prod_{k=1}^{r} \prod_{j^{\prime} \in J(\sigma)}\left(\xi_{j^{\prime}} / \xi_{j_{0}}\right)^{a_{j k} b_{k j^{\prime}}}=\prod_{k=1}^{r} \theta_{x}\left(m_{k}\right)^{a_{j k}}
$$

for $j \in J(\sigma)$, i.e. $x_{\left(\theta_{x}\right)}=x$. Also, from (6) one has

$$
\sum_{j \in J(\sigma)} b_{i j} a_{j k}=\delta_{i k} \quad \text { (Kronecker's delta), }
$$

whence we obtain $\theta_{\left(x_{\theta}\right)}=\theta$. Thus the correspondences $x \mapsto \theta_{x}$ and $\theta \mapsto x_{\theta}$ are mutually inverse, and the correspondences

$$
x \mapsto \theta_{x} \mapsto\left(\theta_{x}\left(m_{1}\right), \ldots, \theta_{x}\left(m_{r}\right)\right)
$$

give the homeomorphisms of the lemma.

4. Next, let $P(\sigma)$ be a projective algebraic set in $P_{\infty}(C)$ defined by the same set of equations ( $\left(2^{\prime}\right)$, but replacing $\Leftrightarrow$ by $\Leftarrow$ in the condition (4). Then we have

Lemma 3. $P(\sigma)=\bigcup_{\tau<\sigma} A_{\tau}$, where $\tau \prec \sigma$ means that $\tau$ is a face of $\sigma$.

To prove $A_{\tau} \subset P(\sigma)$ for $\tau \prec \sigma$, it suffices to show that all the equations $\left(2^{\prime}\right)$ for $\sigma$ hold for $\left(\xi_{j}\right) \in A_{\tau}$. If $c_{j}=0$ for all $j \notin J(\tau)$, this is trivial. Let $\tau=\sigma(\vec{l}), u_{1} \in \vec{l} \subset \Omega, u_{1} \neq 0$, and let $j_{1} \in J(\tau)$. Then, from the relations (2) and (3), one has

$$
\sum_{j \in J(\sigma)} c_{j}\left\langle m^{(j)}-m^{\left(j_{1}\right)}, u_{1}\right\rangle=0
$$

where $\left\langle m^{(j)}-m^{\left(j_{1}\right)}, u_{1}\right\rangle \geqq 0$ for all $j \in J(\sigma)$ and the equality sign holds if 
and only if $j \in J(\tau)$. Hence, if there exists $j \notin J(\tau)$ with $c_{j} \neq 0$, then there also exists $j^{\prime} \notin J(\tau)$ with $c_{j} c_{j^{\prime}}<0$. Therefore, in this case, the equation ( $\left.2^{\prime}\right)$ holds in the form $0=0$.

Next, let $\left(\xi_{j}^{0}\right) \in P(\sigma)$. We put $J_{0}=\left\{j \in J(\sigma) \mid \xi_{j}^{0} \neq 0\right\}$ and let $\tau_{0}$ be the convex closure of $\left\{m^{(j)} \mid j \in J_{0}\right\}$. Then from the equations (2') we can infer that $\tau_{0}$ satisfies the following properties:

(i) $m^{(j)} \in \operatorname{aff}\left(\tau_{0}\right) \cap \sigma \Rightarrow j \in J_{0}$, where $\operatorname{aff}\left(\tau_{0}\right)$ denotes the affine space spanned by $\tau_{0}$.

(ii) If $\tau \prec \sigma$ and if the interior of $\tau$ intersects with $\tau_{0}$, then $\tau \subset \tau_{0}$.

For instance, (ii) can be proved as follows. Let $\left\{m^{\left(j_{1}\right)}, \ldots, m^{\left(j_{t}\right)}\right\}$ be the set of all vertices of $\tau$. Then, under the said assumption, comparing two baricentric expressions of a point in the intersection, one obtains an integral linear relation of the form

$$
\sum_{j \in J_{0}} c_{j} m^{(j)}=\sum_{k=1}^{t} c_{k}^{\prime} m^{\left(j_{k}\right)}
$$

with $c_{j} \geqq 0\left(j \in J_{0}\right)$ and $c_{k}^{\prime}>0(1 \leqq k \leqq t)$. Hence by $\left(2^{\prime}\right)$ one has

$$
\prod_{j \in J_{0}}\left(\xi_{j}^{0}\right)^{c_{j}}=\prod_{k=1}^{t}\left(\xi_{j_{k}}^{0}\right)^{c_{k}^{\prime}}
$$

where the left-hand side is $\neq 0$, so that one also has $\xi_{j_{k}}^{0} \neq 0$ for all $1 \leqq k \leqq t$, i.e. $j_{1}, \ldots, j_{t} \in J_{0}$. Thus $\tau \subset \tau_{0}$.

From the properties (i), (ii), we can conclude immediately that $\tau_{0} \prec \sigma$ and $J_{0}=J\left(\tau_{0}\right)$. Then clearly $\left(\xi_{j}^{0}\right) \in A_{\tau_{0}}$. This completes the proof.

From Lemmas 2 and 3 , we see that $P(\sigma)$ coincides with the (topological) closure of $A_{\sigma}$ and hence is an $r$-dimensional projective variety. Moreover $P(\sigma)$ is rational, for, in the notation of the proof of Lemma 2, the function field of $P(\sigma)$ is a purely transcendental extension of $C$ generated by $\theta_{x}\left(m_{i}\right)(1 \leqq i \leqq r)$.

REMARK. Through the isomorphism of Lemma 2, the torus group $\left(C^{\times}\right)^{r}$ acts on $A_{\sigma}$, and it is not difficult to see that this action can naturally be extended to $P(\sigma)$. Thus $\left(C^{\times}\right)^{r} \rightarrow P(\sigma)$ is an "equivariant projective imbedding of tori" in the sense of Mumford [5] except that $P(\sigma)$ may not be normal.

5. The above construction of the varieties $A_{\sigma}$ and $P(\sigma)$ can be extended to the case of an arbitrary convex polygon $\sigma$ in $U$ spanned by finitely many lattice points, if we take up a priori only those linear relations (2) satisfying (3), which may be called "affine" relations. Then all the above results (except Lemma 1) remain true. It is an interesting combinatorial question to find, for a given $\sigma$, simple "fundamental relations" from among these integral affine relations (2). Especially, one may ask whether 
or not the binomial relations of the form

$$
m^{\left(j_{0}\right)}+m^{\left(j_{2}\right)}=m^{\left(j_{1}\right)}+m^{\left(j_{3}\right)} \quad\left(j_{0}, j_{1}, j_{2}, j_{3} \in J(\sigma)\right)
$$

are fundamental. When this condition is satisfied, we call $\sigma$ "good"; in that case, $P(\sigma)$ is defined by a set of quadratic equations of the form $\xi_{j_{0}} \xi_{j_{2}}=\xi_{j_{1}} \xi_{j_{3}}$. Clearly all 1-dimensional polygons are "good". Yamaguchi $[10]$ proved that any 2-dimensional convex polygon spanned by lattice points is "good" except for the case of triangles such that there exists at least one lattice point in their interior but there exist no lattice points on their sides except vertices (Figure 1).

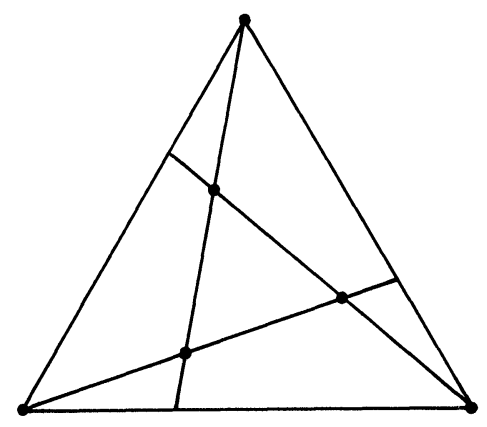

Figure 1

For such exceptional triangles, a set of fundamental relations is obtained by adding certain trinomial affine relations to the binomial ones. Furthermore, he showed that for $n=3$ all cells $\sigma$ on the Hariko are "good".

6. Now let $u_{0} \in U_{c}, u_{1} \in \Omega$ and put

$$
u(\lambda)=u_{0}+i \lambda u_{1}, \quad \lambda \in \boldsymbol{R} .
$$

Then, for a sufficiently large $\lambda$, we have $u(\lambda) \in U+i \Omega$. It is clear that one has $\zeta^{(j)}(u(\lambda)) \rightarrow 0(\lambda \rightarrow \infty)$ for all $j$ and that, if $j_{1} \in J(\sigma)\left(\sigma=\sigma(\vec{l}), u_{1} \in \vec{l}\right)$, then

$$
\zeta^{(j)}(u(\lambda)) / \zeta^{\left(j_{1}\right)}(u(\lambda)) \begin{cases}=\zeta^{(j)}\left(u_{0}\right) / \zeta^{\left(j_{1}\right)}\left(u_{0}\right) & \text { for } j \in J(\sigma), \\ \rightarrow 0 & \text { for } j \notin J(\sigma) .\end{cases}
$$

Therefore we have $\lim _{\lambda \rightarrow \infty} \phi(u(\lambda)) \in A_{\sigma}$, and actually, by Lemma 2 , it is easy to see that all points in $A_{\sigma}$ are obtained as limit points of this type. We put

$$
\begin{aligned}
\mathscr{U} & =\phi(U+i \Omega) \cup \mathscr{U}_{\infty}, \\
\mathscr{U}_{\infty} & =\left\{\lim _{\lambda \rightarrow \infty} \phi(u(\lambda)) \mid u_{0} \in U_{c}, u_{1} \in \Omega\right\} .
\end{aligned}
$$


Then we have

$$
\mathscr{U}_{\infty}=\bigcup_{\sigma \in \Sigma} A_{\sigma}=\bigcup_{\sigma \in \Sigma}^{\prime} P(\sigma),
$$

where, by Lemma 3, one may restrict $\sigma$ to the $(n-1)$-dimensional cells in the second union, denoted by $U^{\prime}$.

Moreover the unions in (9) are locally finite. To see this, we note that, for any $x_{0}=\left(\xi_{j}^{0}\right) \in P_{\infty}(C)$ and for any finite set of indices $J$ such that $\xi_{j}^{0} \neq 0$ for all $j \in J$, there exists a neighbourhood $N$ of $x_{0}$ such that for any $\left(\xi_{j}\right) \in N$ one has $\xi_{j} \neq 0$ for all $j \in J$. It follows that if $x_{0} \in A_{\sigma}$ then there exists a neighbourhood $N$ of $x_{0}$ such that $\mathscr{U}_{\infty} \cap N \subset \bigcup_{\tau \succ \sigma} A_{\tau}$, which proves our assertion. Similarly, if $x_{0} \in \phi(U+i \Omega)$ then (taking $J$ such that $J \notin J(\sigma)$ for any $\sigma \in \Sigma)$ we find a neighbourhood $N$ of $x_{0}$ such that $\mathscr{U}_{\infty} \cap N=\varnothing$, which shows that $\phi(U+i \Omega)$ is relatively open in $\mathscr{U}$. We have thus proved the following theorem.

THEOREM 1. $\mathscr{U}_{\infty}$ is relatively closed in $\mathscr{U}$ and is a locally finite union of the $(n-1)$-dimensional rational projective varieties $P(\sigma)(\sigma \in \Sigma, \operatorname{dim} \sigma=$ $n-1)$. Moreover, one has

$$
P(\sigma) \cap P(\tau)=P(\sigma \cap \tau) \quad \text { for } \sigma, \tau \in \Sigma,
$$

where we put $P(\varnothing)=\varnothing$.

The last assertion is an immediate consequence of Lemma 3. Thus the configuration of $\mathscr{U}_{\infty}$ is completely determined by the Hariko.

7. In order to study the structure of $\mathscr{U}$ more closely, let $\left\{m_{1}, \ldots, m_{s}\right\}$ be a finite subset of $M^{*}$ and put

$$
\begin{aligned}
\Lambda & =\left\{u \in U \mid\left\langle m_{i}, u\right\rangle>0(1 \leqq i \leqq s)\right\}, \\
\Lambda^{*} & =\left\{\sum_{i=1}^{s} \lambda_{i} m_{i} \mid \lambda_{i}>0\right\} .
\end{aligned}
$$

We suppose that $\Lambda \neq \varnothing$, and $\bar{\Lambda}-\{0\} \subset \Omega$. Then $\Lambda$ and $\Lambda^{*}$ are mutually dual open (convex) polyhedral cones, and one has $\bar{\Omega}-\{0\} \subset \Lambda^{*}$. We assume further that $\left\{m_{1}, \ldots, m_{s}\right\}$ generates the semigroup $\bar{\Lambda}^{*} \cap M^{*}-\{0\}$. Thus $\left\{m_{1}, \ldots, m_{s}\right\}$ contains a basis of $M^{*}$.

We define a partial ordering in $\Omega \cap M^{*}$ by

$$
m^{(j)}<_{\Lambda} m^{\left(j^{\prime}\right)} \Leftrightarrow m^{\left(j^{\prime}\right)}-m^{(j)} \in \bar{\Lambda}^{*}-\{0\} .
$$

A point $m^{\left(j_{1}\right)}$ is called $\Lambda$-minimal if there exist no $m^{(j)} \in \Omega \cap M^{*}$ such that $m^{(j)}<_{\Lambda} m^{\left(j_{1}\right)}$. It is clear that for any $j_{0}$ the set of all $m^{(j)}$ which are not $>_{\Lambda} m^{\left(j_{0}\right)}$ is finite; in particular, the set of all $\Lambda$-minimal elements in 
$\Omega \cap M^{*}$ is finite. We denote by $J(\Lambda)$ the set of all $j$ such that $m^{(j)}$ is $\Lambda$-minimal.

Now for a positive integer $s$ we put

$$
\begin{aligned}
D_{s} & =\left\{\left(\zeta_{i}\right) \in C^{s}|| \zeta_{i} \mid<1(1 \leqq i \leqq s)\right\}, \\
D_{s}^{\times} & =\left\{\left(\zeta_{i}\right) \in C^{s}|0<| \zeta_{i} \mid<1(1 \leqq i \leqq s)\right\},
\end{aligned}
$$

and consider these as open subsets of an $s$-dimensional complex projective space $P_{s}(\boldsymbol{C})$, whose coordinates are given by $\left(\zeta_{0}, \zeta_{1}, \ldots, \zeta_{s}\right)$. On the other hand, for any (nonempty) subset $J$ of the set of indices $N=\{1,2, \ldots\}$, we denote by $P_{J}(C)$ a complex projective space of dimension $\operatorname{Card}(J)-1$ whose coordinates are given by $\left(\xi_{j}\right)(j \in J)$. We define an algebraic set $Q_{\Lambda, J}$ in $P_{s}(\boldsymbol{C}) \times P_{J}(\boldsymbol{C})$ as follows. For each pair $j_{1}, j_{2} \in J$ we write

$$
m^{\left(j_{2}\right)}-m^{\left(j_{1}\right)}=\sum_{i=1}^{s} a_{i} m_{i} \quad\left(a_{i} \in Z\right) .
$$

$Q_{\Lambda, J}$ is by definition the set of all points $\left(\zeta_{i}\right) \times\left(\xi_{j}\right) \in P_{s}(\boldsymbol{C}) \times P_{J}(\boldsymbol{C})$ satisfying all the equations

$$
\left(\prod_{+} \zeta_{i}^{a_{i}}\right) \xi_{j_{1}}=\left(\prod_{-} \zeta_{i}^{\left|a_{i}\right|}\right) \xi_{j_{2}}
$$

corresponding to the linear relations of the form (10), where we put $a_{0}=-\sum_{i=1}^{s} a_{i}$ and as before $\prod_{+}\left(\right.$resp. $\left.\prod_{-}\right)$denotes the product taken over all $i(0 \leqq i \leqq s)$ such that $a_{i}>0$ (resp. $\left.a_{i}<0\right)$. Note that, as a special case of the equations $\left(10^{\prime}\right)$, we have an equation of the form

$$
\prod_{+} \zeta_{i}^{a_{i}}=\prod_{-} \zeta_{i}^{\left|a_{i}\right|}
$$

whenever we have an integral linear relation $\sum_{i=1}^{s} a_{i} m_{i}=0$.

We denote by $\pi=\pi_{s, J}$ and $\pi^{\prime}=\pi_{s, J}^{\prime}$ the projections of $P_{s}(C) \times P_{J}(C)$ onto $P_{s}(C)$ and $P_{J}(C)$, respectively, and put

$$
D_{\Lambda, J}=Q_{\Lambda, J} \cap \pi^{-1}\left(D_{s}\right), \quad D_{\Lambda, J}^{\times}=Q_{\Lambda, J} \cap \pi^{-1}\left(D_{s}^{\times}\right) .
$$

$D_{\Lambda, J}$ is an algebraic set in $D_{s} \times P_{J}(C)$ defined by the set of equations $\left(10^{\prime}\right)$, where we put $\zeta_{0}=1$. It is then clear that the map

$$
U+i \Lambda \ni u \stackrel{\phi_{\Lambda, J}}{\longrightarrow}\left(\zeta_{i}(u)\right) \times\left(\zeta^{(j)}(u)\right) \in D_{s}^{\times} \times P_{J}(C),
$$

where $\zeta_{i}$ stands for $\zeta_{m_{i}}$, gives rise to a homeomorphism

$$
(U+i \Lambda) / M \approx D_{\Lambda, J}^{\times} .
$$

Note that the definitions of $D_{\Lambda, J}, \phi_{\Lambda, J}, \ldots$ depend not only on $\Lambda$ but on the choice of the (ordered) set of generators $\left\{m_{i}\right\}$ of the semigroup $\bar{\Lambda}^{*} \cap M^{*}-\{0\}$. 
If $J \subset J^{\prime} \subset N$, we have a natural projection map

$$
p_{J J^{\prime}}: P_{s}(C) \times P_{J^{\prime}}(C) \rightarrow P_{s}(C) \times P_{J}(C) .
$$

LEMMA 4. If $J(\Lambda) \subset J \subset J^{\prime}$, the projection $p_{J J^{\prime}}$ induces a homeomorphism of $D_{\Lambda, J^{\prime}}$ onto $D_{\Lambda, J}$, which is a birational analytic isomorphism if $J$ and $J^{\prime}$ are finite.

Proof. For any $k \in J^{\prime}$ there exists $j_{1} \in J(\Lambda) \subset J$ such that $m^{\left(j_{1}\right)} \leqq_{\Lambda} m^{(k)}$, i.e.

$$
m^{(k)}-m^{\left(j_{1}\right)}=\sum_{i=1}^{s} b_{i} m_{i}
$$

with $b_{i} \in \boldsymbol{Z}, b_{i} \geqq 0$. Therefore we have

$$
\xi_{k}=\left(\prod_{i} \zeta_{i}^{b_{i}}\right) \xi_{j_{1}}
$$

in $D_{\Lambda, J^{\prime}}$, which shows that the rational map $p_{J^{\prime}}$ is everywhere defined and injective on $D_{\Lambda, J^{\prime}}$. Clearly we have $p_{J J^{\prime}}\left(D_{\Lambda, J^{\prime}}\right) \subset D_{\Lambda, J}$. Now let $\left(\zeta_{i}\right) \times\left(\xi_{j}\right) \in D_{\Lambda, J}$. Then, by virtue of the equations $\left(10^{\prime}\right)$ holding in $D_{\Lambda, J}$, the expression on the right-hand side of $\left(12^{\prime}\right)$ does not depend on the choice of $j_{1} \in J$ nor on the expression (12). Hence we can define a polynomial map

$$
q_{J^{\prime} J}: D_{\Lambda, J} \rightarrow D_{s} \times P_{J^{\prime}}(C)
$$

by $\left(12^{\prime}\right)$ such that $p_{J J^{\prime}} \circ q_{J^{\prime} J}=\mathrm{id}$. To show that the image of $q_{J^{\prime} J}$ is actually contained in $D_{\Lambda, J^{\prime}}$, let $k, k^{\prime} \in J^{\prime}$ and $m^{\left(k^{\prime}\right)}-m^{(k)}=\sum_{i=1}^{s} a_{i}^{\prime} m_{i}$. We choose $j_{1} \in J$ as above and similarly $j_{2} \in J$ such that

$$
m^{\left(k^{\prime}\right)}-m^{\left(j_{2}\right)}=\sum_{i=1}^{s} b_{i}^{\prime} m_{i}
$$

with $b_{i}^{\prime} \in Z, b_{i}^{\prime} \geqq 0$. We have to show that the $\xi_{k}$ and $\xi_{k^{\prime}}$ defined by the equations of the form (12') satisfy the equation

$$
\left(\prod_{+}^{\prime} \zeta_{i}^{a_{i}^{\prime}}\right) \xi_{k}=\left(\prod_{-}^{\prime} \zeta_{i}^{\left|a_{i}^{\prime}\right|}\right) \xi_{k^{\prime}}
$$

Put $a_{i}=a_{i}^{\prime}+b_{i}-b_{i}^{\prime}(1 \leqq i \leqq s)$. Then we have $m^{\left(j_{2}\right)}-m^{\left(j_{1}\right)}=\sum_{i=1}^{s} a_{i} m_{i}$, whence by $\left(10^{\prime}\right)$ holding in $D_{\Lambda, J}$

$$
\left(\prod_{+} \zeta_{i}^{a_{i}}\right) \xi_{j_{1}}=\left(\prod_{-} \zeta_{i}^{\left|a_{i}\right|}\right) \xi_{j_{2}} .
$$

If we put $c_{i}=\operatorname{Max}\left(a_{i}^{\prime}, 0\right)+b_{i}-\operatorname{Max}\left(a_{i}, 0\right)$, then $c_{i}$ is always $\geqq 0$ and, multiplying $\prod_{i} \zeta_{i}^{c_{i}}$ on both sides of $(*)$, we get $(* *)$. Q.E.D. 
We write $D(\Lambda), D^{\times}(\Lambda), \phi_{\Lambda}, \pi_{\Lambda}^{\prime}, \ldots$ for $D_{\Lambda, N}, D_{\Lambda, N}^{\times}, \phi_{\Lambda, N}, \pi_{s, N}^{\prime}, \ldots$, respectively. Then by the above lemma we have

$$
D(\Lambda) \approx D_{\Lambda, J}, \quad D^{\times}(\Lambda) \approx D_{\Lambda, J}^{\times}
$$

for any $J \supset J(\Lambda)$. Taking $J$ to be finite, we see that $D(\Lambda)$ has a (welldefined) structure of an analytic space isomorphic to an open subset (in the sense of usual topology) of a finite-dimensional projective algebraic set, and $D^{\times}(\Lambda)$ is a Zariski-open subset of $D(\Lambda)$. We call $D^{\circ}(\Lambda)$ the (topological) closure of $D^{\times}(\Lambda)$ in $D(\Lambda)$. Then $D^{\circ}(\Lambda)$ is an irreducible component of $D(\Lambda)$, which is isomorphic to an open subset of an $n$ dimensional projective variety (determined uniquely up to birational equivalence). From the definitions we have

$$
\phi \mid(U+i \Lambda)=\pi_{\Lambda}^{\prime} \circ \phi_{\Lambda}, \quad \phi_{\Lambda}(U+i \Lambda)=D^{\times}(\Lambda) .
$$

8. Let $\left\{m_{1}^{\prime}, \ldots, m_{t}^{\prime}\right\}$ be another finite subset of $M^{*}$ satisfying the conditions stated at the beginning of $\S 7$, and let $\Lambda^{\prime}$ be the corresponding open polyhedral cone (i.e. $\left\{m_{1}^{\prime}, \ldots, m_{t}^{\prime}\right\}$ generates $\overline{\Lambda^{\prime *}} \cap M^{*}-\{0\}$ ). Suppose that $\Lambda \subset \Lambda^{\prime}$. Then one has

$$
m_{i^{\prime}}^{\prime}=\sum_{i=1}^{s} d_{i i^{\prime}} m_{i}
$$

with $d_{i i^{\prime}} \in \boldsymbol{Z}, d_{i i^{\prime}} \geqq 0$. For $\left(\zeta_{i}\right) \times\left(\xi_{j}\right) \in D(\Lambda)$, define

where

$$
\phi_{\Lambda^{\prime} \Lambda}\left(\left(\zeta_{i}\right) \times\left(\xi_{j}\right)\right)=\left(\zeta_{i^{\prime}}^{\prime}\right) \times\left(\xi_{j}\right)
$$

$$
\zeta_{i^{\prime}}^{\prime}=\prod_{i=1}^{s} \zeta_{i}^{d_{i i^{\prime}}} \quad\left(1 \leqq i^{\prime} \leqq t\right) .
$$

Again this is well-defined by the equations $\left(10^{\prime \prime}\right)$ holding in $D(\Lambda)$, and gives a polynomial map of $D(\Lambda)$ into $D_{t} \times P_{\infty}(C)$.

Lemma 5. One has $\phi_{\Lambda^{\prime} \Lambda}(D(\Lambda)) \subset D\left(\Lambda^{\prime}\right)$.

Proof. We have to show that $\left(\zeta_{i}^{\prime}\right) \times\left(\xi_{i}\right) \in D_{t} \times P_{\infty}(C)$ defined by $\left(14^{\prime}\right)$ satisfies all the equations of the form $\left(10^{\prime}\right)$ for $\Lambda^{\prime}$. Let $j_{1}, j_{2} \in N$ and let

$$
m^{\left(j_{2}\right)}-m^{\left(j_{1}\right)}=\sum_{i^{\prime}=1}^{t} a_{i^{\prime}}^{\prime} m_{i^{\prime}}^{\prime}
$$

Then by (14) this is equal to $\sum_{i=1}^{s} a_{i} m_{i}$ where $a_{i}=\sum_{i^{\prime}=1}^{t} d_{i i^{\prime}} a_{i^{\prime}}^{\prime}$. Since $\left(\zeta_{i}\right) \times\left(\xi_{j}\right) \in D(\Lambda)$, one has

$$
\left(\prod_{+} \zeta_{i}^{a_{i}}\right) \xi_{j_{1}}=\left(\prod_{-} \zeta_{i}^{\left|a_{i}\right|}\right) \xi_{j_{2}}
$$


Hence

$$
\left(\prod_{+} \zeta_{i}^{\sum_{+}^{\prime} d_{i i^{\prime}} a_{i^{\prime}}^{\prime}-\sum_{-}^{\prime} d_{i i^{\prime}}\left|a_{i^{\prime}}^{\prime}\right|}\right) \xi_{j_{1}}=\left(\prod_{-} \zeta_{i}^{\sum_{i}^{\prime} d_{i i^{\prime}}\left|a_{i^{\prime}}\right|-\sum_{+}^{\prime} d_{i i^{\prime}} a_{i^{\prime}}^{\prime}}\right) \xi_{j_{2}},
$$

where $\sum_{+}^{\prime}\left(\operatorname{resp} \sum_{-}^{\prime}\right)$ denotes the summation taken over all $i^{\prime}\left(1 \leqq i^{\prime} \leqq t\right)$ such that $a_{i^{\prime}}^{\prime}>0$ (resp. $\left.a_{i^{\prime}}^{\prime}<0\right)$. Multiplying

$$
\prod_{+} \zeta_{i}^{\sum_{i}^{\prime}} d_{i i^{\prime}}\left|a_{i}^{\prime}\right| \cdot \prod_{-} \zeta_{i}^{\sum_{i}^{\prime}} d_{i i^{\prime}} a_{i^{\prime}}^{\prime}
$$

on both sides of $(*)$, one gets

or

$$
\left(\prod_{i=1}^{s} \zeta \sum_{i}^{\prime} d_{i i^{\prime}} a_{i^{\prime}}\right) \xi_{j_{1}}=\left(\prod_{i=1}^{s} \zeta_{i}^{\sum^{\prime}-d_{i i^{\prime}}\left|a_{i^{\prime}}\right|}\right) \xi_{j_{2}}
$$

$$
\left(\prod_{+}^{\prime} \zeta_{i^{\prime}}^{\prime a^{\prime}}\right) \xi_{j_{1}}=\left(\prod_{-}^{\prime} \zeta_{i^{\prime}}^{\prime \prime a^{i^{\prime}}}\right) \xi_{j_{2}}
$$

which proves our assertion. Q.E.D.

From the definitions it is obvious that the following diagram is commutative

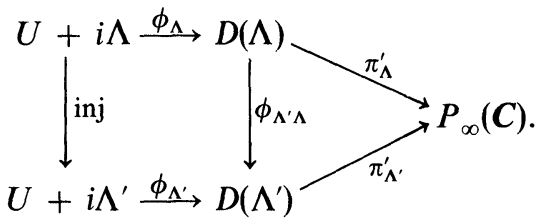

Hence we also have $\phi_{\Lambda^{\prime} \Lambda}\left(D^{0}(\Lambda)\right) \subset D^{0}\left(\Lambda^{\prime}\right)$. It is obvious that, if $\Lambda \subset \Lambda^{\prime} \subset$ $\Lambda^{\prime \prime}(\subset \Omega)$, then we have $\phi_{\Lambda^{\prime \prime} \Lambda^{\prime}} \circ \phi_{\Lambda^{\prime} \Lambda}=\phi_{\Lambda^{\prime \prime} \Lambda}$. In particular, we see that, if $\Lambda=\Lambda^{\prime}, \phi_{\Lambda^{\prime} \Lambda}$ gives an isomorphism of $D(\Lambda)$ onto $D\left(\Lambda^{\prime}\right)$. Thus the structure of $D(\Lambda)$ (and hence that of $D^{0}(\Lambda)$ ) is independent of the choice of the set of generators $\left\{m_{1}, \ldots, m_{s}\right\}$ of the semigroup $\bar{\Lambda}^{*} \cap M^{*}-\{0\}$.

9. To proceed further we need the following notations. For $\sigma \in \Sigma$, put

$$
\Lambda_{\sigma}=\bigcup_{\sigma(\vec{l})=\sigma} \vec{l}
$$

Then $\Lambda_{\sigma}$ is a relatively open, convex polyhedral cone in $U(\sigma)^{\perp}$, where $U(\sigma)$ is the (real) vector space spanned by $M(\sigma)$ and $U(\sigma)^{\perp}$ is the orthogonal complement of $U(\sigma)$ in $U$. Hence we have $\operatorname{dim} \Lambda_{\sigma}=n-\operatorname{dim} \sigma$. It is also clear that

$$
\left\{\begin{aligned}
\sigma \prec \tau & \Rightarrow \Lambda_{\sigma}>\Lambda_{\tau} \\
\Omega & =\bigcup_{\sigma \in \Sigma} \Lambda_{\sigma} \quad \text { (disjoint union). }
\end{aligned}\right.
$$


(Thus $\left\{\Lambda_{\sigma}(\sigma \in \Sigma)\right\}$ is a "rational polyhedral cone decomposition" of $\Omega$ in the sense of Mumford [5], which is dual to our Hariko.) For any open convex cone $\Lambda$ we put

$$
\Sigma_{\Lambda}=\{\sigma(\vec{l}) \mid \vec{l} \subset \Lambda\}=\left\{\sigma \in \Sigma \mid \Lambda_{\sigma} \cap \Lambda \neq \varnothing\right\} .
$$

Then $\Sigma_{\Lambda}$ is a closed (finite) subcomplex of $\Sigma$. In fact, from (16) we have

$$
\bar{\Lambda}_{\sigma}-\{0\}=\bigcup_{\tau \succ \sigma} \Lambda_{\tau}
$$

whence follows that $\sigma \prec \tau, \Lambda_{\tau} \cap \Lambda \neq \varnothing \Rightarrow \Lambda_{\sigma} \cap \Lambda \neq \varnothing$, which proves our assertion.

Lemma 6. For any open polyhedral cone $\Lambda$ as defined in $\S 7, \pi_{\Lambda}^{\prime}\left(D^{0}(\Lambda)\right)$ contains $\phi(U+i \Lambda) \cup \bigcup_{\sigma \in \Sigma_{\Lambda}} A_{\sigma}$ and is contained in

$$
\phi(U+i \Lambda) \cup \bigcup_{\sigma \in \operatorname{star}\left(\Sigma_{\Lambda}\right)} A_{\sigma}
$$

where $\operatorname{star}\left(\Sigma_{\Lambda}\right)=\left\{\tau \in \Sigma \mid \tau \succ \sigma\right.$ for some $\left.\sigma \in \Sigma_{\Lambda}\right\}$.

Proof. To prove the first assertion, let $\sigma \in \Sigma_{\Lambda}, u_{0} \in U_{c}, u_{1} \in \Lambda_{\sigma} \cap \Lambda$, $u_{1} \neq 0$, and put $u(\lambda)=u_{0}+i \lambda u_{1}$. Then $u(\lambda) \in U+i \Lambda$ for sufficiently large $\lambda$. It is clear that one has $\zeta_{i}(u(\lambda)) \rightarrow 0(\lambda \rightarrow \infty)$ for all $1 \leqq i \leqq s$. On the other hand, if we put

$$
\xi_{j}^{0}= \begin{cases}\zeta^{(j)}\left(u_{0}\right) & \text { for } j \in J(\sigma), \\ 0 & \text { for } j \notin J(\sigma),\end{cases}
$$

then $\left(\zeta^{(j)}(u(\lambda))\right) \rightarrow\left(\xi_{j}^{0}\right)$ in $P_{\infty}(C)$. Thus

$$
\lim _{\lambda \rightarrow \infty} \phi_{\Lambda}(u(\lambda))=(0) \times\left(\xi_{j}^{0}\right) \in D^{0}(\Lambda)
$$

and

$$
\pi_{\Lambda}^{\prime}\left((0) \times\left(\xi_{j}^{0}\right)\right)=\left(\xi_{j}^{0}\right) \in A_{\sigma} .
$$

By Lemma 2, any $\left(\xi_{j}^{0}\right) \in A_{\sigma}$ can be written in the form $\left(^{*}\right)$ with some $u_{0} \in U_{C}$. Hence we have $A_{\sigma} \subset \pi_{\Lambda}^{\prime}\left(D^{0}(\Lambda)\right)$.

Next, to prove the second assertion, let $\left\{u^{(v)}(v=1,2, \ldots)\right\}$ be a sequence in $U+i \Lambda$ and suppose that

$$
\phi_{\Lambda}\left(u^{(v)}\right) \rightarrow x_{0}=\left(\zeta_{i}^{0}\right) \times\left(\xi_{j}^{0}\right) \in D^{0}(\Lambda)
$$

when $v \rightarrow \infty$. If $\zeta_{i}^{0} \neq 0$ for all $i$, then clearly $x_{0} \in \phi_{\Lambda}(U+i \Lambda)$. Hence let us assume that $\zeta_{i}^{0}=0$ for some $i$. Then, by taking a suitable subsequence, we may assume that

$$
\begin{aligned}
& u^{(v)}=u_{0}^{(v)}+i \lambda_{v} u_{1}^{(v)}, \\
& u_{0}^{(v)} \rightarrow u_{0} \in U, \quad u_{1}^{(v)} \rightarrow u_{1} \in \bar{\Lambda}-\{0\}, \quad \lambda_{v} \rightarrow \infty,
\end{aligned}
$$

and that $u_{1}^{(v)} \in \Lambda_{\sigma_{0}}(v=1,2, \ldots), u_{1} \in \Lambda_{\sigma}$ for some $\sigma_{0}, \sigma \in \Sigma$. Then one has $\sigma_{0} \in \Sigma_{\Lambda}$ and $\sigma_{0} \prec \sigma$. Put $J_{0}=\left\{j \mid \xi_{j}^{0} \neq 0\right\}$. We contend that 
$J\left(\sigma_{0}\right) \subset J_{0} \subset J(\sigma)$. Take $j_{0} \in J\left(\sigma_{0}\right)$. Then for any $j$

$$
\operatorname{Im}\left(\left\langle m^{(j)}-m^{\left(j_{0}\right)}, u^{(v)}\right\rangle\right)=\lambda_{v}\left\langle m^{(j)}-m^{\left(j_{0}\right)}, u_{1}^{(v)}\right\rangle \geqq 0 .
$$

Hence one has $j_{0} \in J_{0}$, and

$$
j \notin J_{0} \Leftrightarrow \lim _{v \rightarrow \infty}\left(\lambda_{v}\left\langle m^{(j)}-m^{\left(j_{0}\right)}, u_{1}^{(v)}\right\rangle\right)=\infty .
$$

In particular, if $j \notin J(\sigma)$, one has

$$
\lim _{v \rightarrow \infty}\left\langle m^{(j)}-m^{\left(j_{0}\right)}, u_{1}^{(v)}\right\rangle=\left\langle m^{(j)}-m^{\left(j_{0}\right)}, u_{1}\right\rangle>0,
$$

and therefore $j \notin J_{0}$, which proves our contention. Now, for any integral linear relation of the form (2), the corresponding equation $\left(2^{\prime}\right)$ holds for $\left(\zeta^{(j)}\left(u^{(v)}\right)\right)(j \in J(\sigma))$ and hence, going to the limit, the same is also true for $\left(\xi_{j}^{0}\right)$. Therefore one has $\left(\xi_{j}^{0}\right) \in P(\sigma)$. By Lemma 3, this implies $\left(\xi_{j}^{0}\right) \in A_{\tau}$ for some $\tau \prec \sigma$. Thus $J_{0}=J(\tau)$ and we have $\tau \succ \sigma_{0} \in \Sigma_{\Lambda}$, i.e. $\tau \in$ $\operatorname{star}\left(\Sigma_{\Lambda}\right)$. Q.E.D.

10. For each $\sigma \in \Sigma$, we put

$$
\Xi_{\sigma}=\operatorname{star}\left(\Lambda_{\sigma}\right)=\bigcup_{\tau \prec \sigma} \Lambda_{\tau} .
$$

$\Xi_{\sigma}$ is a (not necessarily convex) open polyhedral cone such that $\bar{\Xi}_{\sigma}-\{0\} \subset \Omega$. Let $\left\{m^{(j)} \mid j \in J_{0}(\sigma)\right\}$ be the set of all vertices of $\sigma$ and put $\sigma_{0}^{(j)}=\left\{m^{(j)}\right\} \quad\left(j \in J_{0}(\sigma)\right)$. Then from (16a) and (17) we have $\bar{\Xi}_{\sigma}=\bigcup_{j \in J_{0}(\sigma)} \bar{\Lambda}_{\sigma_{0}^{(j)}}$. It follows that

$$
\Xi_{\sigma}^{*}=\bigcap_{j \in J_{0}(\sigma)} \Lambda_{\sigma_{0}^{(j)}}^{*}
$$

Let $\left\{m_{i}(1 \leqq i \leqq s)\right\}$ be a finite set of generators of the semigroup $\overline{\Xi_{\sigma}^{*}} \cap M^{*}-\{0\}$. We fix one $j_{0} \in J_{0}(\sigma)$. Then, since $\Lambda_{\left.\sigma_{0}^{(j)}\right)}^{*}$ is an open polyhedral cone spanned by $\left\{m^{(k)}-m^{\left(j_{0}\right)}(k \in N)\right\}$, we have for each $1 \leqq i \leqq s$ a (finite) linear relation of the form

$$
a_{i} m_{i}=\sum_{k} a_{i k}\left(m^{(k)}-m^{(j o)}\right)
$$

with $a_{i}, a_{i k} \in Z, a_{i}>0, a_{i k} \geqq 0$. Note that, since $m_{i} \notin U(\sigma)$, there exists for each $i$ at least one $k$ such that $a_{i k}>0$ and $k \notin J(\sigma)$. The convex closure of $\Xi_{\sigma}$ is given by

$$
\Xi_{\sigma}^{* *}=\left\{u \in U \mid\left\langle m_{i}, u\right\rangle>0(1 \leqq i \leqq s)\right\} .
$$

Next we assign to each $\sigma \in \Sigma$ an open set $N_{\sigma}$ in $P_{\infty}(C)$ defined as follows. $N_{\sigma}$ consists of all $\left(\xi_{j}\right) \in P_{\infty}(C)$ satisfying the conditions

$$
\left\{\begin{aligned}
\xi_{j} \neq 0 & \text { for } j \in J(\sigma), \\
\prod_{k}\left|\xi_{k} / \xi_{j 0}\right|^{a_{i k}}<1 & \text { for } 1 \leqq i \leqq s .
\end{aligned}\right.
$$


Then it is immediate that we have

$$
\left\{\begin{array}{c}
\phi(U+i \Omega) \cap N_{\sigma}=\phi\left(U+i \Xi_{\sigma}^{* *}\right), \\
A_{\sigma} \subset \mathscr{U}_{\infty} \cap N_{\sigma} \subset \bigcup_{\tau>\sigma} A_{\tau} .
\end{array}\right.
$$

Since $\left\{\Xi_{\sigma}^{* *}(\sigma \in \Sigma)\right\}$ is a star-finite open covering of $\Omega$, this implies that $\left\{\mathscr{U} \cap N_{\sigma}(\sigma \in \Sigma)\right\}$ is a star-finite open covering of $\mathscr{U}$.

LEMMA 7. We have

$$
\mathscr{U} \cap N_{\sigma}=\pi^{\prime}\left(D^{0}\left(\Xi_{\sigma}^{* *}\right)\right) \cap N_{\sigma} .
$$

Proof. By Lemma 6 and (20), it suffices to show that $A_{\tau} \cap N_{\sigma} \subset$ $\pi^{\prime}\left(D^{0}\left(\Xi_{\sigma}^{* *}\right)\right)$ for $\tau \succ \sigma, \tau \neq \sigma$. Changing the order of the indices $i$, we may assume that for $1 \leqq i \leqq s$

$$
m_{i} \in U(\tau) \Leftrightarrow 1 \leqq i \leqq s_{1} .
$$

Then, since $\Lambda_{\tau}$ is a face of $\Xi_{\sigma}$, one has

$$
\Lambda_{\tau} \subset\left\{u \in U(\tau)^{\perp} \mid\left\langle m_{i}, u\right\rangle>0 \text { for } s_{1}+1 \leqq i \leqq s\right\} .
$$

Let $u_{1} \in \Lambda_{\tau}$ and $u_{0} \in U_{C}$ be such that $\left\langle m_{i}, \operatorname{Im} u_{0}\right\rangle>0$ for $1 \leqq i \leqq s_{1}$. Then $u(\lambda)=u_{0}+i \lambda u_{1} \in \Xi_{\sigma}^{* *}$ for sufficiently large $\lambda$, and one has

where

$$
\lim _{\lambda \rightarrow \infty} \phi_{\Xi_{\sigma}^{* *}}(u(\lambda))=\left(\zeta_{i}^{0}\right) \times\left(\xi_{j}^{0}\right) \in D^{0}\left(\Xi_{\sigma}^{* *}\right),
$$

$$
\zeta_{i}^{0}=\left\{\begin{array}{ll}
\zeta_{i}\left(u_{0}\right) & \left(1 \leqq i \leqq s_{1}\right) \\
0 & \left(s_{1}+1 \leqq i \leqq s\right)
\end{array} \text { and } \quad \xi_{j}^{0}= \begin{cases}\zeta^{(j)}\left(u_{0}\right) & (j \in J(\tau)) \\
0 & (j \notin J(\tau)) .\end{cases}\right.
$$

From the relation (18) and from our choice of $u_{0}$, we have

$$
\prod_{k}\left|\xi_{k}^{0} / \xi_{j_{0}}^{0}\right|^{a_{i k}}=\left|\zeta_{i}^{0}\right|^{a_{i}}<1
$$

for all $1 \leqq i \leqq s$. Thus we see that the condition (19) is satisfied for $\left(\xi_{j}^{0}\right)$, i.e. $\left(\xi_{j}^{0}\right) \in N_{\sigma}$. By Lemma 2 , it is clear that any $\left(\xi_{j}^{0}\right) \in A_{\tau} \cap N_{\sigma}$ can be obtained in this manner. Q.E.D.

It follows from this lemma that $\mathscr{U} \cap N_{\sigma}$ does not depend on the choice of the set of generators $\left\{m_{i}(1 \leqq i \leqq s)\right\}$ nor on the relation (18).

LEMMA 8. $\mathscr{U} \cap N_{\sigma}$ has a structure of an analytic space such that, for any sufficiently large finite set $J \subset N$, the natural projection $P_{\infty}(C) \rightarrow$ $P_{J}(C)$ induces an analytic isomorphism of $\mathscr{U} \cap N_{\sigma}$ onto an open subset of a projective variety in $P_{J}(C)$ whose birational class is uniquely determined. ${ }^{3}$

\footnotetext{
${ }^{3}$ By a result in [5], $\mathscr{U} \cap N_{\sigma}$ is normal, if $J\left(\Xi_{\sigma}^{* *}\right)=J(\sigma)$ and if we can take $a_{i}=1$ for all $1 \leqq i \leqq s$ in (18) where $j_{0}$ may vary in $J(\sigma)$.

It is not known if $\mathscr{U}$ is always normal.
} 
Proof. Let $J$ be a finite subset of $N$ containing $J\left(\Xi_{\sigma}^{* *}\right)(\supset J(\sigma))$ and all $k$ such that $a_{i k}>0$ for some $1 \leqq i \leqq s$, and let $N_{\sigma, J}$ be the image of $N_{\sigma}$ under the projection map $P_{\infty}(\boldsymbol{C}) \rightarrow P_{J}(\boldsymbol{C})$, which is everywhere defined on $N_{\sigma}$; then $N_{\sigma, J}$ is an open subset of $P_{J}(\boldsymbol{C})$. For simplicity we write $Q_{\sigma, J}, D_{\sigma, J}, \ldots$ for $Q_{\Xi_{\alpha}^{* *, J}}, D_{\Xi^{* *, J}}, \ldots$, respectively. Let $D_{\sigma, J}^{0}$ denote the (topological) closure of $D_{\sigma, J}^{\times}$in $D_{\sigma, J}$; then $D_{\sigma, J}^{0}\left(\approx D^{0}\left(\Xi_{\sigma}^{* *}\right)\right)$ is an irreducible component of $D_{\sigma, J}\left(\approx D\left(\Xi_{\sigma}^{* *}\right)\right)$. Moreover there exists a unique $n$-dimensional (global) irreducible component $Q_{\sigma, J}^{0}$ of $Q_{\sigma, J}$ such that

$$
D_{\sigma, J}^{0}=Q_{\sigma, J}^{0} \cap D_{\sigma, J} .
$$

Now, for $\left(\zeta_{i}\right) \times\left(\xi_{j}\right) \in Q_{\sigma, J}^{0} \cap\left(\pi^{\prime}\right)^{-1}\left(N_{\sigma, J}\right)$ with $\zeta_{0} \neq 0$, we have by (18)

$$
\left(\zeta_{i} / \zeta_{0}\right)^{a_{i}}=\prod_{k}\left(\xi_{k} / \xi_{j_{0}}\right)^{a_{i k}} \quad(1 \leqq i \leqq s) .
$$

Hence we have by $(19)\left|\zeta_{i} / \zeta_{0}\right|<1$ for all $1 \leqq i \leqq s$, i.e. $\left(\zeta_{i}\right) \times\left(\xi_{j}\right) \in D_{\sigma, J}^{0}$. This shows that $D_{\sigma, J}^{0} \cap\left(\pi^{\prime}\right)^{-1}\left(N_{\sigma, J}\right)$ is a Zariski-open subset of $Q_{\sigma, J}^{0} \cap$ $\left(\pi^{\prime}\right)^{-1}\left(N_{\sigma, J}\right)$. Therefore $\pi^{\prime}\left(D_{\sigma, J}^{0}\right) \cap N_{\sigma, J}$ is an open subset of $\pi^{\prime}\left(Q_{\sigma, J}^{0}\right) \cap N_{\sigma, J}$. Thus $\pi^{\prime}\left(D_{\sigma, J}^{0}\right) \cap N_{\sigma, J}$ is an open subset of a projective variety $\pi^{\prime}\left(Q_{\sigma, J}^{0}\right)$ in $P_{J}(C)$.

By $\left(18^{\prime}\right)$ we see that the projection map $\pi^{\prime}=\pi_{\sigma, J}^{\prime}$ induces a proper analytic map (of finite degree) of $D_{\sigma, J}^{0} \cap\left(\pi^{\prime}\right)^{-1}\left(N_{\sigma, J}\right)$ onto $\pi^{\prime}\left(D_{\sigma, J}^{0}\right) \cap N_{\sigma, J}$. In view of Lemma 4, we see that, if $J \subset J^{\prime}$, the natural projection $P_{J J^{\prime}}$ induces a (birational) analytic isomorphism of $D_{\sigma, J^{\prime}}^{0}$ onto $D_{\sigma, J}^{0}$ which makes the following diagram commutative.

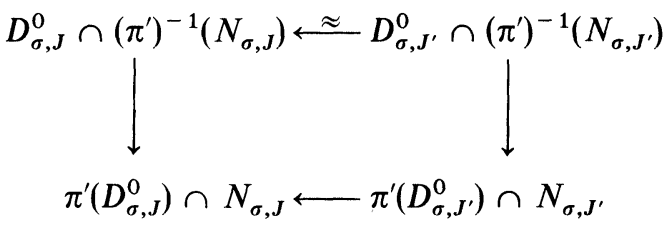

It follows that, if $J$ is sufficiently large, the natural projection $P_{\infty}(C) \rightarrow$ $P_{J}(C)$ induces a homeomorphism

$$
\pi^{\prime}\left(D^{0}\left(\Xi_{\sigma}^{* *}\right)\right) \cap N_{\sigma} \approx \pi^{\prime}\left(D_{\sigma, J}^{0}\right) \cap N_{\sigma, J},
$$

which defines a structure of an analytic space on $\pi^{\prime}\left(D^{0}\left(\Xi_{\sigma}^{* *}\right)\right) \cap N_{\sigma}$ independently of the choice of $J$. As we mentioned above this analytic space is isomorphic to an open subset of an $n$-dimensional projective variety whose birational class is uniquely determined. In view of Lemma 7 , the proof is complete.

We note that, when $\mathscr{U} \cap N_{\sigma} \cap N_{\tau} \neq \varnothing$, the analytic structures on $\mathscr{U} \cap N_{\sigma} \cap N_{\tau}$ induced from those on $\mathscr{U} \cap N_{\sigma}$ and $\mathscr{U} \cap N_{\tau}$ coincide. In fact, by the above lemma, if $J$ is sufficiently large, the natural projection $P_{\infty}(C) \rightarrow P_{J}(C)$ induces analytic isomorphisms

$$
\mathscr{U} \cap N_{\sigma} \approx \pi^{\prime}\left(D_{\sigma, J}^{0}\right) \cap N_{\sigma, J}, \quad \mathscr{U} \cap N_{\tau} \approx \pi^{\prime}\left(D_{\tau, J}^{0}\right) \cap N_{\tau, J} .
$$


Hence, if $\mathscr{U} \cap N_{\sigma} \cap N_{\tau} \neq \varnothing$, we have

$$
\pi^{\prime}\left(D_{\sigma, J}^{0}\right) \cap N_{\sigma, J} \cap N_{\tau, J}=\pi^{\prime}\left(D_{\tau, J}^{0}\right) \cap N_{\sigma, J} \cap N_{\tau, J} \neq \varnothing,
$$

which implies $\pi^{\prime}\left(Q_{\sigma, J}^{0}\right)=\pi^{\prime}\left(Q_{\tau, J}^{0}\right)$. This proves our assertion.

Since $\mathscr{U}$ is a star-finite union of $\left\{\mathscr{U} \cap N_{\sigma}(\sigma \in \Sigma)\right\}$, we can conclude that $\mathscr{U}$ is locally compact and has a structure of an $n$-dimensional irreducible analytic space. Actually, as we have seen above, $\mathscr{U}$ is an analytic space obtained by gluing together open subsets of projective varieties by everywhere biregular birational correspondences; we will express this by saying that $\mathscr{U}$ is "locally algebraic". From the definition of the analytic structure on $\mathscr{U}$, it is clear that $\mathscr{U}_{\infty}$ is an $(n-1)$-dimensional closed analytic subspace of $\mathscr{U}$ and $\mathscr{U}-\mathscr{U}_{\infty}$ is analytically isomorphic to the quotient space $(U+i \Omega) / M$.

11. Now a natural question arises. Is the analytic space $\mathscr{U}$ (or its normalization) nonsingular? Unfortunately, the answer is negative in general. ${ }^{4}$ But, from what we mentioned in $\$ 10, \mathscr{U}$ is nonsingular at $x_{0} \in \mathscr{U}_{\infty} \cap N_{\sigma}$, if $D^{0}\left(\Xi_{\sigma}^{* *}\right)$ is nonsingular at $y_{0} \in\left(\pi^{\prime}\right)^{-1}\left(x_{0}\right)$ and if the projection $\pi^{\prime}$ is a local analytic isomorphism at $y_{0}$. We shall give here a simple sufficient condition for these.

Suppose that there are $n+1$ points $m^{\left(i_{0}\right)}, \ldots, m^{\left(i_{n}\right)}$ on the Hariko $|\Sigma|$ such that $m_{k}=m^{\left(i_{k}\right)}-m^{\left(i_{0}\right)}(1 \leqq k \leqq n)$ form a basis of $M^{*}$ and that $\Lambda_{\sigma_{0}}^{*}\left(\sigma_{0}=\left\{m^{\left(i_{0}\right)}\right\}\right)$ is spanned by $\left\{m_{k}(1 \leqq k \leqq n)\right\}$. We contend that under this assumption all points on $\pi^{\prime}\left(D^{0}\left(\Lambda_{\sigma_{0}}\right)\right)$ are nonsingular.

In fact, by the assumption, every $m^{(j)} \in \Omega \cap M^{*}$ can be written uniquely in the form

$$
m^{(j)}=m^{\left(i_{0}\right)}+\sum_{k=1}^{n} a_{k} m_{k}
$$

with $a_{k} \in \boldsymbol{Z}, a_{k} \geqq 0$. Hence we have

$$
\xi_{j}=\left(\prod_{k=1}^{n} \zeta_{k}^{a_{k}}\right) \xi_{i_{0}}
$$

in $D\left(\Lambda_{\sigma}\right)$; in particular, $\xi_{i_{k}}=\zeta_{k} \xi_{i_{0}}$. Therefore the natural projection $D_{n} \times P_{\infty}(C) \rightarrow D_{n}$ induces an isomorphism of $D\left(\Lambda_{\sigma_{0}}\right)$ onto $D_{n}$. It follows that $D^{0}\left(\Lambda_{\sigma_{0}}\right)=D\left(\Lambda_{\sigma_{0}}\right)$ and $D^{0}\left(\Lambda_{\sigma_{0}}\right)$ is nonsingular. It is also obvious that the projection $D_{n} \times P_{\infty}(C) \rightarrow P_{\infty}(C)$ induces an isomorphism of $D^{0}\left(\Lambda_{\sigma_{0}}\right)$ onto $\pi^{\prime}\left(D^{0}\left(\Lambda_{\sigma_{0}}\right)\right)$. Thus $\pi^{\prime}\left(D^{0}\left(\Lambda_{\sigma_{0}}\right)\right)$ is nonsingular. Note that, in this case, one has $\Xi_{\sigma_{0}}=\Lambda_{\sigma_{0}}$ and by Lemmas 6 and $7 \mathscr{U} \cap N_{\sigma_{0}}=\pi^{\prime}\left(D^{0}\left(\Lambda_{\sigma_{0}}\right)\right)$.

\footnotetext{
${ }^{4}$ ADDED IN PROOF. For instance, in the case of Hilbert modular surface, it can be shown that our blow-up $\mathscr{U}$ is obtained from the nonsingular surface $Y^{+}$constructed in Hirzebruch [3b] by blowing down each chain of curves $S_{k}$ with self-intersection number -2 to a point. The singularities thus created are just (isolated) ramification points, and so $\mathscr{U}$ has a structure of $V$-manifold.
} 
12. Finally, we define a natural action of $\Gamma$ on $\mathscr{U}$. First, since $\Omega$ and $M^{*}$ are both invariant under ${ }^{t} \Gamma$, so are $\Omega \cap M^{*}, \Sigma=\Sigma\left(\Omega, M^{*}\right), \ldots$ It is clear from the definition that for a half-line $\vec{l} \subset \Omega$ one has

$$
\sigma(\gamma(\vec{l}))={ }^{t} \gamma^{-1}(\sigma(\vec{l}))
$$

Hence we have $\gamma\left(\Lambda_{\sigma}\right)=\Lambda_{t_{\gamma}{ }^{-1} \sigma}, \gamma\left(\Xi_{\sigma}\right)=\Xi_{t_{\gamma-1} \sigma}$.

We define an action of $\Gamma$ on the set of indices $N$ by

$$
j^{\prime}=\gamma j \Leftrightarrow m^{\left(j^{\prime}\right)}={ }^{t} \gamma^{-1} m^{(j)},
$$

and its action on $P_{\infty}(C)$ by

$$
\left(\xi_{j}^{\prime}\right)=\gamma\left(\xi_{j}\right) \Leftrightarrow \xi_{j}^{\prime}=\xi_{\gamma^{-1} j} .
$$

Then, since $\left\langle m^{(j)}, \gamma u\right\rangle=\left\langle{ }^{t} \gamma m^{(j)}, u\right\rangle=\left\langle m^{\left(\gamma^{-1} j\right)}, u\right\rangle$, we have

$$
\phi(\gamma u)=\gamma \phi(u) \quad \text { for } u \in U+i \Omega,
$$

i.e. the map $\phi$ is $\Gamma$-equivariant. It follows that $\mathscr{U}_{\infty}$ is $\Gamma$-invariant, and in view of $(23)$ we have

$$
\gamma P(\sigma)=P\left({ }^{t} \gamma^{-1} \sigma\right) .
$$

Now let $N_{\sigma}$ be as defined in $\S 10$ and suppose that $N_{t_{\gamma}{ }^{-1} \sigma}$ is defined with respect to the set of generators $\left\{\gamma^{t} m_{i}(1 \leqq i \leqq s)\right\}$ and the relations corresponding to (18). Then it is clear that $\gamma\left(N_{\sigma}\right)=N_{t_{\gamma}-1_{\sigma}}$ and $\gamma$ induces a (birational) analytic isomorphism of $\mathscr{U} \cap N_{\sigma}$ onto $\mathscr{U} \cap N_{t_{\gamma}-1}$. We express this property of $\gamma$ by saying that $\gamma$ is a "locally birational" analytic automorphism of $\mathscr{U}$.

Now, from the reduction theory for $\Gamma[2]$, we know that there exists a finite number of $(n-1)$-dimensional cells $\sigma_{v}\left(1 \leqq v \leqq v_{0}\right)$ such that every $(n-1)$-dimensional cell $\sigma$ in $\Sigma$ is ${ }^{t} \Gamma$-equivalent to a unique $\sigma_{v}$. Then we have

$$
\Omega=\bigcup_{1 \leqq \nu \leqq v_{0}, \gamma \in \Gamma} \Xi_{t_{\gamma}{ }^{*}{ }_{\sigma_{v}}}^{* *}=\bigcup_{1 \leqq \nu \leqq v_{0}, \gamma \in \Gamma} \gamma \Xi_{\sigma_{v}}^{* *},
$$

which is a star-finite union. It follows from (20) that we have

$$
\mathscr{U}=\bigcup_{\gamma, v}\left(\mathscr{U} \cap N_{t_{\gamma^{-1}} \sigma_{v}}\right)=\bigcup_{\gamma, v} \gamma\left(\mathscr{U} \cap N_{\sigma_{v}}\right) .
$$

Since this is a star-finite open covering of $\mathscr{U}$, we see that the action of $\Gamma$ on $\mathscr{U}$ is properly discontinuous. Taking the quotient with respect to $\Gamma$, we obtain from (28)

$$
\begin{aligned}
\Gamma \backslash \mathscr{U} & \approx(\tilde{\Gamma} \backslash(U+i \Omega)) \cup \Gamma \backslash \mathscr{U}_{\infty} \\
& =\bigcup_{v=1}^{v_{0}} \Gamma \backslash \Gamma\left(\mathscr{U} \cap N_{\sigma_{v}}\right) .
\end{aligned}
$$


By a theorem of $\mathrm{H}$. Cartan, $\Gamma \backslash \mathscr{U}$ is an analytic space. Moreover, if we put $\Gamma_{\sigma}=\{\gamma \in \Gamma \mid \gamma P(\sigma)=P(\sigma)\}$, then $\Gamma_{\sigma}$ is a finite group and we have

$$
\Gamma \backslash \mathscr{U}_{\infty} \approx \bigcup_{\nu=1}^{v_{0}} \Gamma_{\sigma_{v}} \backslash P\left(\sigma_{v}\right)
$$

Thus $\Gamma \backslash \mathscr{U}_{\infty}$, which is a closed analytic subspace of $\Gamma \backslash \mathscr{U}$, is a finite union of $(n-1)$-dimensional projective varieties.

Summing up, we have obtained the following result.

THEOREM 2. $\mathscr{U}=\phi(U+i \Omega) \cup \mathscr{U}_{\infty}$ carries a natural structure of an $n$-dimensional, irreducible, locally algebraic analytic space, in which $\mathscr{U}_{\infty}$ is a closed analytic subspace and $\phi(U+i \Omega)$ is an open subspace isomorphic to the quotient space $(U+i \Omega) / M$. The group $\Gamma$ acts on $\mathscr{U}$ as a properly discontinuous group of locally birational analytic automorphisms, and the quotient space $\Gamma \backslash \mathscr{U}$ is a finite union of quotient spaces of open subsets of projective varieties by birational equivalence relations. Moreover, $\mathscr{U}_{\infty}$ is stable under $\Gamma$ and the quotient space $\Gamma \backslash \mathscr{U}_{\infty}$ is a finite union of $(n-1)-$ dimensional projective varieties of the form $\Gamma_{\sigma} \backslash P(\sigma)$, where $\Gamma_{\sigma}$ is a finite subgroup of $\Gamma$ which stabilizes $P(\sigma)$.

\section{REFERENCES}

1. W. L. Baily and A. Borel, Compactification of arithmetic quotients of bounded symmetric domains, Ann. of Math. (2) 84 (1966), 442-528. MR 35 \# 6870.

2. A. Borel and Harish-Chandra, Arithmetic subgroups of algebraic groups, Ann. of Math. (2) 75 (1962), 485-535. MR 26 \#5081.

3a. F. Hirzebruch, The Hilbert modular group, resolution of the singularities at the cusp and related problems, Sém. Bourbaki, 23-e année, 1970/71, n 396.

3b. - - Hilbert modular surfaces. Enseignement Math. (to appear).

4. J.-I. Igusa, A desingularization problem in the theory of Siegel modular functions, Math. Ann. 168 (1967), 228-260. MR 36 \#1439.

5. D. Mumford, et al., Toroidal embeddings, I, Seminar notes at Harvard Univ., 1972.

6. I. I. Pjateckiř-Shapiro, Geometry of classical domains and automorphic functions, Fizmatgiz, Moscow, 1961; English transl., Automorphic functions and the geometry of classical domains, Math. and its Applications, vol. 8, Gordon and Breach, New York, 1969. MR 25 \#231; 40 \#5908.

7a. I. Satake, Compactifications of the quotient spaces for arithmetically defined discontinuous groups, Ann. of Math. (2) 72 (1960), 555-580. MR 30 \# 594.

7b. - , Realization of symmetric domains as Siegel domain of the third kind, Lecture Notes, Univ. of California, 1972.

8. C. L. Siegel, Zur Theorie der Modulfunktionen n-ten Grades, Comm. Pure Appl. Math. 8 (1955), 677-681. MR 17, 602.

9. E. B. Vinberg, The theory of convex homogeneous cones, Trudy Moskov. Mat. Obšc 12 (1963), 303-358 = Trans. Moscow Math. Soc., 1963, 340-403. MR 28 \#1637.

10. H. Yamaguchi, On defining equations of certain projective varieties (to appear).

Department of Mathematics, University of California, Berkeley, California 94720 